ELECTRONIC RESEARCH ANNOUNCEMENTS OF THE AMERICAN MATHEMATICAL SOCIETY

Volume 11, Pages 1-11 (January 14, 2005)

S $1079-6762(05) 00141-1$

\title{
ON GLUING FORMULAS FOR THE SPECTRAL INVARIANTS OF DIRAC TYPE OPERATORS
}

\author{
PAUL LOYA AND JINSUNG PARK
}

(Communicated by Michael Taylor)

\begin{abstract}
In this note, we announce gluing and comparison formulas for the spectral invariants of Dirac type operators on compact manifolds and manifolds with cylindrical ends. We also explain the central ideas in their proofs.
\end{abstract}

\section{THE GLUING PROBLEM FOR THE SPECTRAL INVARIANTS}

Since their inception, the eta invariant and the $\zeta$-determinant of Dirac type operators have influenced mathematics and physics in innumerable ways. Especially with the development of quantum field theory, the behavior of these spectral invariants under gluing of the underlying manifold has become an increasingly important topic. However, the gluing formula for the $\zeta$-determinant of a Dirac Laplacian has remained an open question due to the nonlocal nature of this invariant. In fact, Bleecker and Booss-Bavnbek stated that [2, p. 89] "no precise pasting formulas are obtained but only adiabatic ones." In [23, 24, we give precise gluing formulas for $\zeta$-determinants of Dirac type operators on compact manifolds and manifolds with cylindrical ends, respectively, and moreover we present new and unified derivations of the gluing formulas for both invariants. The purpose of this note is to announce these gluing formulas for the spectral invariants and to indicate the main ideas in their proofs. We also announce a relative invariant formula proved in [25, 24].

We begin with describing the gluing problem for compact manifolds. Let $\mathcal{D}$ be a Dirac type operator acting on $C^{\infty}(M, S)$ where $M$ is a closed compact Riemannian manifold of arbitrary dimension and $S$ is a Clifford bundle over $M$. Let $Y$ be an embedded hypersurface in $M$ and let $M=M_{-} \cup M_{+}$be decomposition of $M$ into manifolds with boundary such that $\partial M_{-}=\partial M_{+}=Y$. We assume that all geometric structures are of product type over a tubular neighborhood $N=$ $[-1,1] \times Y$ of $Y$ where the Dirac operator takes the product form $\mathcal{D}=G\left(\partial_{u}+D_{Y}\right)$, where $G$ is a unitary operator on $S_{0}:=\left.S\right|_{Y}$ and $D_{Y}$ is a Dirac type operator over $Y$ satisfying $G^{2}=-\operatorname{Id}$ and $D_{Y} G=-G D_{Y}$. Recall that the eta function of $\mathcal{D}$ and the zeta function of $\mathcal{D}^{2}$ are defined through the heat operator $e^{-t \mathcal{D}^{2}}$ via

$$
\eta_{\mathcal{D}}(s)=\frac{1}{\Gamma\left(\frac{s+1}{2}\right)}\left(\int_{0}^{1}+\int_{1}^{\infty}\right) t^{\frac{s-1}{2}} \operatorname{Tr}\left(\mathcal{D} e^{-t \mathcal{D}^{2}}\right) d t,
$$

Received by the editors October 6, 2004

2000 Mathematics Subject Classification. Primary 58J28, 58J52.

(C)2005 American Mathematical Society 


$$
\zeta_{\mathcal{D}^{2}}(s)=\frac{1}{\Gamma(s)}\left(\int_{0}^{1}+\int_{1}^{\infty}\right) t^{s-1} \operatorname{Tr}\left(e^{-t \mathcal{D}^{2}}\right) d t,
$$

where the first integrals in (1.1) and (1.2) are defined a priori for Re $s \gg 0$ and the second ones for Re $s \ll 0$, and both extend to meromorphic functions on $\mathbb{C}$ that are regular at $s=0$; actually, the second integral in (1.1) is entire, but we present these general definitions because they work later for $b$-spectral invariants on manifolds with cylindrical ends (see Section 2). The eta invariant $\eta(\mathcal{D})$ is by definition the value $\eta_{\mathcal{D}}(0)$, the reduced eta invariant is $\tilde{\eta}(\mathcal{D}):=(\eta(\mathcal{D})+\operatorname{dim} \operatorname{ker}(\mathcal{D})) / 2$, and the $\zeta$-determinant is by definition

$$
\operatorname{det}_{\zeta} \mathcal{D}^{2}:=\exp \left(-\left.\frac{d}{d s}\right|_{s=0} \zeta_{\mathcal{D}^{2}}(s)\right)
$$

The eta invariant was introduced in the paper [1] by Atiyah, Patodi, and Singer as the boundary correction term in their index formula for manifolds with boundary. The $\zeta$-determinant was introduced by Ray and Singer in the paper [36] on the analytic torsion. Since their introductions, these invariants have impacted geometry, topology, and physics in incredible ways; cf. Singer [40, 41].

By restriction, $\mathcal{D}$ induces Dirac type operators $\mathcal{D}_{+}$over $M_{+}$and $\mathcal{D}_{-}$over $M_{-}$. For these operators, we choose orthogonal projections $\mathcal{P}_{+}, \mathcal{P}_{-}$over $L^{2}\left(Y, S_{0}\right)$ that provide us with well-posed boundary conditions for $\mathcal{D}_{+}, \mathcal{D}_{-}$in the sense of Seeley [39]. Then the operators

$$
\mathcal{D}_{\mathcal{P}_{ \pm}}: \operatorname{dom}\left(\mathcal{D}_{\mathcal{P}_{ \pm}}\right) \rightarrow L^{2}\left(M_{ \pm}, S\right)
$$

where

$$
\operatorname{dom}\left(\mathcal{D}_{\mathcal{P}_{ \pm}}\right):=\left\{\phi \in H^{1}\left(M_{ \pm}, S\right) \mid \mathcal{P}_{ \pm}\left(\left.\phi\right|_{Y}\right)=0\right\},
$$

share many of the analytic properties of $\mathcal{D}$; in particular, they are Fredholm and have discrete spectra, but are not necessarily self-adjoint. Amongst such projectors are the (orthogonalized) Calderón projectors $\mathcal{C}_{ \pm}$[7], which are projectors defined intrinsically as the unique orthogonal projectors onto the closures in $L^{2}\left(Y, S_{0}\right)$ of the infinite-dimensional Cauchy data spaces of $\mathcal{D}_{ \pm}$:

$$
\left\{\left.\phi\right|_{Y} \mid \phi \in C^{\infty}\left(M_{ \pm}, S\right), \mathcal{D}_{ \pm} \phi=0\right\} \subset C^{\infty}\left(Y, S_{0}\right) .
$$

In order to have well-defined eta invariants and $\zeta$-determinants, it is necessary to restrict to a subclass of projectors. A natural class is formed by those in the smooth, self-adjoint Grassmannian $G r_{\infty}^{*}\left(\mathcal{D}_{ \pm}\right)$, which consists of orthogonal projections $\mathcal{P}_{ \pm}$ such that $\mathcal{P}_{ \pm}-\mathcal{C}_{ \pm}$are smoothing operators and $G \mathcal{P}_{ \pm}=\left(\mathrm{Id}-\mathcal{P}_{ \pm}\right) G$. Examples of such projectors are the generalized APS spectral projectors $\mathcal{P}_{-}=\Pi_{<}+\frac{1+\sigma_{1}}{2} \Pi_{0}$, $\mathcal{P}_{+}=\Pi_{>}+\frac{1+\sigma_{2}}{2} \Pi_{0}$, where $\Pi_{<}, \Pi_{>}, \Pi_{0}$ are the orthogonal projectors onto the negative, positive, and zero eigenspaces of $D_{Y}$, respectively, and the $\sigma_{i}$ 's are involutions on $\operatorname{ker}\left(D_{Y}\right)$ anticommuting with $G$. For $\mathcal{P}_{ \pm} \in G r_{\infty}^{*}\left(\mathcal{D}_{ \pm}\right)$, the invariants $\eta\left(\mathcal{D}_{\mathcal{P}_{ \pm}}\right)$and $\operatorname{det}_{\zeta} \mathcal{D}_{\mathcal{P}_{ \pm}}^{2}$ can be defined via the formulas (1.1) and (1.2); see Grubb [12, 13, Loya and Park [25, and Wojciechowski [44.

We now have all the ingredients to state the gluing problem. The gluing problem is to describe the "defects"

$$
\frac{\operatorname{det}_{\zeta} \mathcal{D}^{2}}{\operatorname{det}_{\zeta} \mathcal{D}_{\mathcal{P}_{+}}^{2} \cdot \operatorname{det}_{\zeta} \mathcal{D}_{\mathcal{P}_{-}}^{2}}=?, \quad \tilde{\eta}(\mathcal{D})-\tilde{\eta}\left(\mathcal{D}_{\mathcal{P}_{+}}\right)-\tilde{\eta}\left(\mathcal{D}_{\mathcal{P}_{-}}\right)=?
$$

in terms of recognizable data. The gluing problem for the eta invariant has been solved by several authors. Moreover, because the variation of the eta invariant is 
local, a variety of formulas and proofs have appeared (many modulo $\mathbb{Z}$ ); see, for instance, Brüning and Lesch [3, Bunke [5], Dai and Freed [9], Hassell, Mazzeo, and Melrose [16], Kirk and Lesch [18], Mazzeo and Melrose [28], Müller [32], Park and Wojciechowski [35, Wojciechowski [43, 44]; cf. the superb survey articles by Mazzeo and Piazza 29] or Bleecker and Booss-Bavnbek 22. Because of the highly nonlocal nature of the $\zeta$-determinant and its variation, according to Bleecker and Booss-Bavnbek [2, p. 90] the $\zeta$-determinants (of a Dirac operator and its square) are "the most subtle and the most fascinating objects of our study." Nonetheless, the gluing problem for the $\zeta$-determinant of Laplace type operators with local boundary conditions was solved by Burghelea, Friedlander, and Kappeler [6] and has been further extended by Carron 8], Hassell [14, Hassell and Zelditch [15], Lee [19, Loya and Park [22, Vishik [42], and many others. However, the gluing formula for the $\zeta$-determinant of a Dirac Laplacian has remained an open question, partly due to the nonlocal nature of the $\zeta$-determinant and its variation and the technical aspects inherent in the global pseudodifferential boundary problems required for Dirac type operators. We now describe our solution to the gluing problem.

To state our main theorem, we recall that the Calderón projectors $\mathcal{C}_{ \pm}$have the matrix forms

$$
\mathcal{C}_{ \pm}=\frac{1}{2}\left(\begin{array}{cc}
\operatorname{Id} & \kappa_{ \pm}^{-1} \\
\kappa_{ \pm} & \operatorname{Id}
\end{array}\right)
$$

with respect to the decomposition $L^{2}\left(Y, S_{0}\right)=L^{2}\left(Y, S^{+}\right) \oplus L^{2}\left(Y, S^{-}\right)$, where $S^{ \pm} \subset$ $S_{0}$ are the $( \pm i)$-eigenspaces of $G$. The maps $\kappa_{ \pm}: L^{2}\left(Y, S^{+}\right) \rightarrow L^{2}\left(Y, S^{-}\right)$are isometries, so that $U:=-\kappa_{-} \kappa_{+}^{-1}$ is a unitary operator over $L^{2}\left(Y, S^{-}\right)$, which is moreover of Fredholm determinant class. We denote by $\widehat{U}$ the restriction of $U$ to the orthogonal complement of its (-1)-eigenspace. We also put

$$
\mathcal{L}:=\sum_{k=1}^{h_{M}} \gamma_{0} U_{k} \otimes \gamma_{0} U_{k}=\sum_{k=1}^{h_{M}}\left\langle\cdot, \gamma_{0} U_{k}\right\rangle_{L^{2}\left(Y, S_{0}\right)} \gamma_{0} U_{k},
$$

where $h_{M}=\operatorname{dim} \operatorname{ker}(\mathcal{D}), \gamma_{0}$ is the restriction map from $M$ to $Y$, and $\left\{U_{k}\right\}$ is an orthonormal basis of $\operatorname{ker}(\mathcal{D})$. Then $\mathcal{L}$ is a positive operator on the finite-dimensional vector space $\gamma_{0}(\operatorname{ker}(\mathcal{D}))$. The following theorem is the main result of [23].

Theorem 1.1 ([23]). The following gluing formulas hold:

$$
\begin{gathered}
\frac{\operatorname{det}_{\zeta} \mathcal{D}^{2}}{\operatorname{det}_{\zeta} \mathcal{D}_{\mathcal{C}_{+}}^{2} \cdot \operatorname{det}_{\zeta} \mathcal{D}_{\mathcal{C}_{-}}^{2}}=2^{-\zeta_{D_{Y}^{2}}(0)-h_{Y}}(\operatorname{det} \mathcal{L})^{-2} \operatorname{det}_{F}\left(\frac{2 \operatorname{Id}+\widehat{U}+\widehat{U}^{-1}}{4}\right), \\
\tilde{\eta}(\mathcal{D})-\tilde{\eta}\left(\mathcal{D}_{\mathcal{C}_{+}}\right)-\tilde{\eta}\left(\mathcal{D}_{\mathcal{C}_{-}}\right)=\frac{1}{2 \pi i} \log \operatorname{det}_{F} U(\bmod \mathbb{Z}),
\end{gathered}
$$

where $h_{Y}=\operatorname{dim} \operatorname{ker}\left(D_{Y}\right)$, $\operatorname{det}_{F}$ denotes the Fredholm determinant, Log is the principal value of the logarithm, and the integer defect in the eta formula is given in terms of the winding numbers of the function $\operatorname{det}_{F}\left(K(\lambda) K^{c}(\lambda)^{-1}\right)$ appearing in Section 4

Let us remark that the $\zeta$-determinant formula in Theorem 1.1 is a new result, and only its adiabatic limit form [34], [35] has been proved hitherto. The formulation of the integer defect in the eta formula in Theorem 1.1 is also new. The eta formula, without integer ambiguity, in terms of $\operatorname{Tr} \log U$ was proved by Kirk and Lesch [18] Th. 5.10] using spectral flow, the Scott-Wojciechowski comparison theorem [38], 
and the rotating boundary condition technique from Brüning and Lesch [3]. Our proof of the eta formula is different from and independent of their proof, and is obtained "simultaneously" as a byproduct from the proof for the $\zeta$-determinant.

We can generalize the gluing formulas in Theorem 1.1 in terms of other boundary conditions by using Theorem 3.1 (see Section 3) proved in [25]. Let $\mathcal{P}_{1} \in G r_{\infty}^{*}\left(\mathcal{D}_{-}\right)$ and $\mathcal{P}_{2} \in G r_{\infty}^{*}\left(\mathcal{D}_{+}\right)$. Then $\mathcal{P}_{1}$ and $\mathcal{P}_{2}$ determine maps $\kappa_{1}$ and $\kappa_{2}$ as in (1.4), and we can define $U_{1}:=\kappa_{-} \kappa_{1}^{-1}, U_{2}:=\kappa_{2} \kappa_{+}^{-1}$ and $U_{12}:=-\kappa_{1} \kappa_{2}^{-1}$ over $L^{2}\left(Y, S^{-}\right)$. As before, we let $\widehat{U}_{i}$ denote the restriction of $U_{i}$ to the orthogonal complement of its $(-1)$-eigenspace. We define the operator $\mathcal{L}_{1}$ over the finite-dimensional vector space $\operatorname{Im}\left(\mathcal{C}_{-}\right) \cap \operatorname{Im}\left(\operatorname{Id}-\mathcal{P}_{1}\right)$ by

$$
\mathcal{L}_{1}=-P_{1} G \mathcal{R}_{-}^{-1} G P_{1},
$$

where $\mathcal{R}_{-}$is the sum of the Dirichlet to Neumann maps on the double of $M_{-}$, which was introduced by Burghelea, Friedlander, and Kappeler in [6], and $P_{1}$ is the orthogonal projection onto $\operatorname{Im}\left(\mathcal{C}_{-}\right) \cap \operatorname{Im}\left(\operatorname{Id}-\mathcal{P}_{1}\right)$. Then $\mathcal{L}_{1}$ is a positive operator [25]. We define $\mathcal{L}_{2}$ in a similar way. We can now state the general gluing formulas for the spectral invariants.

Theorem 1.2. The following general gluing formulas hold:

$$
\begin{gathered}
\frac{\operatorname{det}_{\zeta} \mathcal{D}^{2}}{\operatorname{det}_{\zeta} \mathcal{D}_{\mathcal{P}_{1}}^{2} \cdot \operatorname{det}_{\zeta} \mathcal{D}_{\mathcal{P}_{2}}^{2}}= \\
\times 2^{-\zeta_{D_{Y}^{2}}(0)-h_{Y}}(\operatorname{det} \mathcal{L})^{-2} \operatorname{det}_{F}\left(\frac{2 \operatorname{Id}+\widehat{U}+\widehat{U}^{-1}}{4}\right) \\
\left.\tilde{\eta}(\mathcal{D})-\tilde{\eta}\left(\mathcal{D}_{\mathcal{P}_{1}}\right)-\tilde{\eta}\left(\mathcal{D}_{\mathcal{P}_{2}}\right)=\frac{1}{2 \pi i} \operatorname{Let} \mathcal{L}_{i}\right)^{-2} \cdot \operatorname{det}_{F}\left(\frac{2 \mathrm{Id}+\widehat{U}_{i}+\widehat{U}_{i}^{-1}}{4}\right)^{-1},
\end{gathered}
$$

where the integer defect in the eta formula can be identified exactly in terms of winding numbers of specific naturally defined operators.

Let us remark that the adiabatic decomposition formulas presented in [34], [35], which are proved mainly using the Duhamel principle and scattering theory, can be derived from the $\zeta$-determinant formula in Theorem 1.2] (cf. [27]).

\section{The GLUiNG PROBLEM ON MANIFOLDS WITH CYLINDRICAL ENDS}

We now describe the solution to the gluing problem on manifolds with cylindrical ends presented in [24. Assume now that instead of $M_{+}$being compact, it is a noncompact half-infinite cylinder: $M_{+}=[0, \infty) \times Y$, over which $\mathcal{D}$ is of product type. We still assume that $M_{-}$is compact. Then $M$ is called a manifold with cylindrical end. For a noncompact manifold $M$ with cylindrical end, the heat operators $\mathcal{D} e^{-t \mathcal{D}^{2}}$ and $e^{-t \mathcal{D}^{2}}$ are not of trace class. In particular, the definitions (1.1) and (1.2) cannot be used as in the compact case. There are two principal ways to make sense of these invariants. One way is to define so-called relative invariants as in Bruneau [4], Carron [8, Müller [33], and others, whereby we subtract off certain operators that make the difference of the heat operators of trace class, and the other way is to use Melrose's $b$-trace [30], ${ }^{b} \mathrm{Tr}$, which is a natural substitute for the trace. In particular, $\mathcal{D} e^{-t \mathcal{D}^{2}}$ and $e^{-t \mathcal{D}^{2}}$ are $b$-trace class. Moreover, ${ }^{b} \operatorname{Tr}\left(\mathcal{D} e^{-t \mathcal{D}^{2}}\right)$ and ${ }^{b} \operatorname{Tr}\left(e^{-t \mathcal{D}^{2}}\right)$ have asymptotic expansions in half-integer powers of $t$ as $t \rightarrow 0$ and $t \rightarrow \infty$. It follows that the $b$-eta function ${ }^{b} \eta_{\mathcal{D}}(s)$ and the ${ }^{b} \zeta$-function ${ }^{b} \zeta_{\mathcal{D}^{2}}(s)$ can 
be defined exactly as in formulas (1.1) and (1.2), respectively, where we replace $\operatorname{Tr}$ with ${ }^{b} \mathrm{Tr}$. Furthermore, ${ }^{b} \eta_{\mathcal{D}}(s)$ and ${ }^{b} \zeta_{\mathcal{D}^{2}}(s)$ extend to define meromorphic functions on $\mathbb{C}$ that are regular at $s=0$, so we can define the $b$-eta invariant of $\mathcal{D}$ by ${ }^{b} \eta(\mathcal{D}):={ }^{b} \eta_{\mathcal{D}}(0)$, and the $b$-determinant of $\mathcal{D}^{2}$ by $\operatorname{det}_{b} \mathcal{D}^{2}:=\exp \left(-{ }^{b} \zeta_{\mathcal{D}^{2}}^{\prime}(0)\right)$.

In order to discuss the gluing formula for the $b$-spectral invariants into the decomposition $M=M_{-} \cup M_{+}$, we need to impose a boundary condition on $M_{+}$. Note that $M_{-}$is compact so $\mathcal{D}_{\mathcal{C}_{-}}$is defined as before. Hence, we need a "Calderón projector" on the noncompact manifold $M_{+}$, which is found by looking at the Cauchy data space of $\mathcal{D}$ over the whole manifold $M$ :

$$
\left\{\left.\phi\right|_{Y} \mid \phi \in C^{\infty}(M, S), \mathcal{D} \phi=0\right\} \subset C^{\infty}\left(Y, S_{0}\right) .
$$

Atiyah, Patodi, and Singer [1] (cf. 30, 31) showed that an element $\psi$ of this space is the restriction $\psi=\left.\phi\right|_{Y}$ of an $L^{2}$ section $\phi$ over $M$ if and only if $\left(\Pi_{<}+\frac{1+\sigma}{2} \Pi_{0}\right) \psi=$ 0 , where $\sigma$ is the unitary map on $\operatorname{ker}\left(D_{Y}\right)$ such that $\sigma^{2}=\operatorname{Id}$ and $\sigma G=-G \sigma$ determined by the scattering matrix. For this reason, the natural projection on $M_{+}$ is

$$
\mathcal{C}_{+}:=\Pi_{>}+\frac{1-\sigma}{2} \Pi_{0}
$$

We can now define $\mathcal{D}_{\mathcal{C}_{+}}$exactly as before (see (1.3)) in the case when $M_{+}$was compact. However, since $M_{+}$is a manifold with cylindrical end, we need to use the $b$-trace to define the corresponding $b$-spectral invariants, ${ }^{b} \eta\left(\mathcal{D}_{\mathcal{C}_{+}}\right)$and $\operatorname{det}_{b} \mathcal{D}_{\mathcal{C}_{+}}^{2}$.

We now have all the ingredients to state the gluing problem for manifolds with cylindrical end. The (b-)gluing problem is to describe the "defects"

$$
\frac{\operatorname{det}_{b \zeta} \mathcal{D}^{2}}{\operatorname{det}_{\zeta} \mathcal{D}_{\mathcal{C}_{-}}^{2} \cdot \operatorname{det}_{b} \mathcal{D}_{\mathcal{C}_{+}}^{2}}=?, \quad{ }^{b} \tilde{\eta}(\mathcal{D})-\tilde{\eta}\left(\mathcal{D}_{\mathcal{C}_{-}}\right)-{ }^{b} \tilde{\eta}\left(\mathcal{D}_{\mathcal{C}_{+}}\right)=?
$$

in terms of recognizable data. Just as before, the projectors $\mathcal{C}_{ \pm}$can be written as (1.4) for unitary operators $\kappa_{ \pm}$, and we denote by $\widehat{U}$ the restriction of $U:=-\kappa_{-} \kappa_{+}^{-1}$ to the orthogonal complement of its (-1)-eigenspace. Also, we define $\mathcal{L}$ as in (1.5), using the orthonormal basis $\left\{U_{k}\right\}$ of $\operatorname{ker}_{L^{2}}(\mathcal{D})$. Then $\mathcal{L}$ is a positive operator on the finite-dimensional vector space $\gamma_{0}\left(\operatorname{ker}_{L^{2}}(\mathcal{D})\right)$. The following theorem is proved in 24 .

Theorem 2.1 (24]). The following gluing formulas hold:

$$
\begin{aligned}
& \frac{\operatorname{det}_{b \zeta} \mathcal{D}^{2}}{\operatorname{det}_{\zeta} \mathcal{D}_{\mathcal{C}_{-}}^{2} \cdot \operatorname{det}_{{ }_{\zeta}} \mathcal{D}_{\mathcal{C}_{+}}^{2}}=2^{-\zeta_{D_{Y}^{2}}(0)-h_{Y}}(\operatorname{det} \mathcal{L})^{-2} \operatorname{det}_{F}\left(\frac{2 \operatorname{Id}+\widehat{U}+\widehat{U}^{-1}}{4}\right), \\
& { }^{b} \tilde{\eta}(\mathcal{D})-\tilde{\eta}\left(\mathcal{D}_{\mathcal{C}_{-}}\right)-{ }^{b} \tilde{\eta}\left(\mathcal{D}_{\mathcal{C}_{+}}\right)={ }^{b} \tilde{\eta}(\mathcal{D})-\tilde{\eta}\left(\mathcal{D}_{\mathcal{C}_{-}}\right)=\frac{1}{2 \pi i} \log \operatorname{det}_{F} U(\bmod \mathbb{Z}),
\end{aligned}
$$

where $h_{Y}:=\operatorname{dim} \operatorname{ker}\left(D_{Y}\right)$ and $\operatorname{det}_{F}$ denotes the Fredholm determinant.

One can show that $\operatorname{det}_{{ }_{\zeta}} \mathcal{D}^{2} / \operatorname{det}_{{ }_{\zeta}} \mathcal{D}_{\mathcal{C}_{+}}^{2}=\operatorname{det}_{\zeta}\left(\mathcal{D}^{2}, \mathcal{D}_{\mathcal{C}_{+}}^{2}\right)$, the relative determinant of the pair $\left(\mathcal{D}^{2}, \mathcal{D}_{\mathcal{C}_{+}}^{2}\right)$, and ${ }^{b} \tilde{\eta}(\mathcal{D})=\tilde{\eta}\left(\mathcal{D}, \mathcal{D}_{\mathcal{C}_{+}}\right)$, the reduced relative eta invariant of the pair $\left(\mathcal{D}, \mathcal{D}_{\mathcal{C}_{+}}\right)$, so this theorem can be written with relative spectral invariants on the left-hand side. Also, some computations show that $\operatorname{det}_{b} \mathcal{D}_{\mathcal{C}_{+}}^{2}=2^{\frac{1}{2} \zeta_{D_{Y}^{2}}(0)}$, so after substituting this expression into our theorem, we can get another version of the $\zeta$-determinant formula without the $\operatorname{det}_{b \zeta} \mathcal{D}_{\mathcal{C}_{+}}^{2}$ term. 


\section{The COMPARISON, OR RELATIVE INVARIANT, PROBLEM}

The comparison problem for the spectral invariants on $M_{-}$can be stated as follows: For $\mathcal{P}_{-} \in G r_{\infty}^{*}\left(\mathcal{D}_{-}\right)$, find formulas for the "defects"

$$
\frac{\operatorname{det}_{\zeta} \mathcal{D}_{\mathcal{P}_{-}}^{2}}{\operatorname{det}_{\zeta} \mathcal{D}_{\mathcal{C}_{-}}^{2}}=?, \quad \tilde{\eta}\left(\mathcal{D}_{\mathcal{P}_{-}}\right)-\tilde{\eta}\left(\mathcal{D}_{\mathcal{C}_{-}}\right)=?
$$

in terms of recognizable data. (Of course, one can consider $M_{+}$too.) For generalized APS spectral projectors, the comparison problem for the eta invariant was first solved modulo $\mathbb{Z}$ by Lesch and Wojciechowski [20 and Müller 31]; later, this integer ambiguity was removed by the first author [21]. For $\mathcal{P}_{-} \in G r_{\infty}^{*}\left(\mathcal{D}_{-}\right)$and assuming the invertibility of $\mathcal{D}_{\mathcal{P}_{-}}$, the comparison problems for the eta invariant and the $\zeta$-determinant were solved by Scott and Wojciechowski [38] and Scott [37]; cf. Forman [10]. The comparison problem for the eta invariant was later solved for $\mathcal{P}_{-} \in G r_{\infty}^{*}\left(\mathcal{D}_{-}\right)$without integer ambiguities by Kirk and Lesch [18] using the Scott-Wojciechowski comparison theorem [38]. In [25], we remove the invertibility assumption on $\mathcal{D}_{\mathcal{P}_{-}}$, and we present a new and unified derivation of the comparison formulas for both the eta invariant and $\zeta$-determinant. For the eta invariant, we obtain a new formulation of the integer defect, and the $\zeta$-determinant formula contains an additional term that is absent in the case of invertible $\mathcal{D}_{\mathcal{P}_{-}}$dealing with $\operatorname{ker}\left(\mathcal{D}_{\mathcal{P}_{-}}\right)$and the Dirichlet to Neumann map on the double of $M_{-}$.

Recall that the Calderón projector $\mathcal{C}_{-}$can be written as (1.4) for a unitary operator $\kappa_{-}$, and $\mathcal{P}_{-}$has a similar decomposition with a unitary operator $\kappa_{\mathcal{P}_{-}}$. We denote by $\widehat{U}_{\mathcal{P}_{-}}$the restriction of $U_{\mathcal{P}_{-}}:=\kappa_{-} \kappa_{\mathcal{P}_{-}}^{-1}$, which is a unitary operator over $L^{2}\left(Y, S^{-}\right)$, to the orthogonal complement of its $(-1)$-eigenspace. We define $\mathcal{L}_{\mathcal{P}_{-}}:=-P_{-} G \mathcal{R}_{-}^{-1} G P_{-}$just as in $(1.6)$, where $P_{-}$is the orthogonal projection onto the finite-dimensional vector space $\operatorname{Im}\left(\mathcal{C}_{-}\right) \cap \operatorname{Im}\left(\operatorname{Id}-\mathcal{P}_{-}\right)$. Then $\mathcal{L}_{\mathcal{P}_{-}}$is a positive operator on this space [25], so $\operatorname{det} \mathcal{L}_{\mathcal{P}_{-}}$is a positive number. The following theorem solves the comparison problem (there is a corresponding formula for the comparison problem on $\left.M_{+}\right)$.

Theorem 3.1 ([25]). The following comparison formulas hold:

$$
\begin{aligned}
& \frac{\operatorname{det}_{\zeta} \mathcal{D}_{\mathcal{P}_{-}}^{2}}{\operatorname{det}_{\zeta} \mathcal{D}_{\mathcal{C}_{-}}^{2}}=\left(\operatorname{det} \mathcal{L}_{\mathcal{P}_{-}}\right)^{2} \cdot \operatorname{det}_{F}\left(\frac{2 \mathrm{Id}+\widehat{U}_{\mathcal{P}_{-}}+\widehat{U}_{\mathcal{P}_{-}}^{-1}}{4}\right), \\
& \tilde{\eta}\left(\mathcal{D}_{\mathcal{P}_{-}}\right)-\tilde{\eta}\left(\mathcal{D}_{\mathcal{C}_{-}}\right)=\frac{1}{2 \pi i} \log \operatorname{det}_{F} U_{\mathcal{P}_{-}}(\bmod \mathbb{Z}),
\end{aligned}
$$

where the integer defect in the eta formula can be identified exactly in terms of winding numbers of a specific naturally defined operator.

Assume now that $M_{+}$is an infinite cylinder as in Section 2 Recall that $\mathcal{C}_{+}:=$ $\Pi_{>}+\frac{1-\sigma}{2} \Pi_{0}($ see $(\underline{2.1}))$ is the natural Calderón projector on the cylinder $M_{+}$. This suggests that the natural boundary projector on $M_{-}$taking into consideration the infinite cylinder should be $\mathcal{S}_{-}:=\Pi_{<}+\frac{1+\sigma}{2} \Pi_{0}$, instead of the Calderón projector $\mathcal{C}_{-}$. The projector $\mathcal{S}_{-}$is called the augmented APS spectral projector (cf. [17]). Then $\mathcal{S}_{-} \in G r_{\infty}^{*}\left(\mathcal{D}_{-}\right)$, so we can define the spectral invariants of $\mathcal{D}_{\mathcal{S}_{-}}$on $M_{-}$. The (b-) comparison problem is to describe the "defects"

$$
\frac{\operatorname{det}_{b} \mathcal{D}^{2}}{\operatorname{det}_{\zeta} \mathcal{D}_{\mathcal{S}_{-}}^{2}}=?, \quad{ }^{b} \eta(\mathcal{D})-\eta\left(\mathcal{D}_{\mathcal{S}_{-}}\right)=?
$$


in terms of recognizable data. Thus, the comparison problem in this context is the comparison of the $b$-spectral invariants on $M$ with those on $M_{-}$corresponding to the "scattering Calderón projector" $\mathcal{S}_{-}$. The following theorem solves the comparison problem on manifolds with cylindrical ends.

Theorem $3.2([24])$. The following comparison formulas hold:

$$
\frac{\operatorname{det}_{b} \mathcal{D}^{2}}{\operatorname{det}_{\zeta} \mathcal{D}_{\mathcal{S}_{-}}^{2}}=2^{-\frac{1}{2} \zeta_{D_{Y}^{2}}(0)-h_{Y}}\left(\frac{\operatorname{det} \mathcal{L}}{\operatorname{det} \mathcal{L}_{-}}\right)^{-2}, \quad{ }^{b} \eta(\mathcal{D})=\eta\left(\mathcal{D}_{\mathcal{S}_{-}}\right)(\bmod 2 \mathbb{Z}),
$$

where $\mathcal{L}_{-}$is defined by (1.5) but with $\left\{U_{k}\right\}$ an orthonormal basis for $\operatorname{ker}\left(\mathcal{D}_{\mathcal{S}_{-}}\right)$.

Thus, the $b$-eta invariant of $\mathcal{D}$ and the eta invariant of $\mathcal{D}_{\mathcal{S}_{-}}$are identical modulo $2 \mathbb{Z}$, while the $\zeta$-determinants differ by data over $Y$ and global data. Because $\zeta$-determinants are highly nonlocal compared to eta invariants, one would not expect the ratio to be unity. This is indeed the case as shown in Theorem 3.2. The $\zeta$-determinant formula is new, and a similar formula for the eta invariants of compatible Dirac operators (without integer ambiguities and with the augmented APS projector $\Pi_{<}+\frac{1-\sigma}{2} \Pi_{0}$ ) was first proved by Müller [31] using a completely different method.

\section{Brief Sketch of the proof of Theorem 1.1}

In this last section, we briefly sketch the proof of Theorem 1.1 we refer to Section 1 for the notation and to our preprint [25] for all the details. We remark that the proofs of Theorems 2.1 and 3.1 are similar in spirit, but the proof of Theorem 2.1 has surprising twists due to the presence of continuous spectrum. For $\lambda$ not in the spectrum of $\mathcal{D}$, the Calderón projectors of $\mathcal{D}_{ \pm}-\lambda$ are defined by

$$
P_{ \pm}(\lambda):= \pm \gamma_{0^{ \pm}}(\mathcal{D}-\lambda)^{-1} \gamma_{0}^{*} G: L^{2}\left(Y, S_{0}\right) \rightarrow L^{2}\left(Y, S_{0}\right),
$$

where $\gamma_{0^{ \pm}}:=\lim _{\varepsilon \rightarrow 0^{ \pm}} \gamma_{\varepsilon}$ with $\gamma_{\varepsilon}$ the restriction map from $M$ to $\{\varepsilon\} \times Y$, and $\gamma_{0}^{*}$ is the adjoint map of $\gamma_{0}$, the restriction to $\{0\} \times Y$. We now introduce an auxiliary model problem over $N=[-1,1] \times Y$. To do so, fix an involution $\omega$ over $\operatorname{ker}\left(D_{Y}\right)$ that anticommutes with $G$. We then impose boundary conditions $\mathcal{C}_{-}^{c}=\Pi_{<}+\frac{1-\omega}{2} \Pi_{0}$ at $\{1\} \times Y, \mathcal{C}_{+}^{c}=\Pi_{>}+\frac{1+\omega}{2} \Pi_{0}$ at $\{-1\} \times Y$. Let us denote the operator $G\left(\partial_{u}+D_{Y}\right)$ with these boundary conditions by $\mathcal{D}^{c}$. Then we can define the Calderón projectors $P_{ \pm}^{c}(\lambda)$ for the restrictions $\mathcal{D}_{ \pm}^{c}-\lambda$ of $\mathcal{D}^{c}-\lambda$ to $M_{ \pm} \cap N$ exactly as in 4.1). Let $V$ be a unitary operator on $L^{2}\left(Y, S_{0}\right)$ that satisfies $V\left(\operatorname{Id}-\mathcal{C}_{+}\right) V^{-1}=\mathcal{C}_{-}$. Then we define operators $K(\lambda)$ and $K^{c}(\lambda)$ on $L^{2}\left(Y, S_{0}\right)$ by defining their inverses as

$$
\begin{aligned}
K(\lambda)^{-1} & =\mathcal{C}_{+} P_{+}(\lambda)+\left(\operatorname{Id}-\mathcal{C}_{+}\right) V^{-1} \mathcal{C}_{-} P_{-}(\lambda), \\
K^{c}(\lambda)^{-1} & =\mathcal{C}_{+}^{c} P_{+}^{c}(\lambda)+\mathcal{C}_{-}^{c} P_{-}^{c}(\lambda) .
\end{aligned}
$$

(The operators on the right-hand side do turn out to be invertible.) From these formulas, we see that $K(\lambda)$ and $K^{c}(\lambda)$ "link" the Calderón projectors of $\mathcal{D}_{ \pm}-\lambda$ and $\mathcal{D}_{ \pm}^{c}-\lambda$, respectively. Moreover, these operators have quite remarkable properties as we shall see. First, $K(\lambda) K^{c}(\lambda)^{-1}$ is of Fredholm determinant class, so $\operatorname{det}_{F}\left(K(\lambda) K^{c}(\lambda)^{-1}\right)$ makes sense. We henceforth fix a simply connected region of the plane consisting of the upper and lower half-planes and an interval on the real axis and fix a corresponding logarithm $\log \operatorname{det}_{F}\left(K(\lambda) K^{c}(\lambda)^{-1}\right)$ depending holomorphically on $\lambda$ in this region. Let us define $\mathcal{D}_{\mathcal{C}}=\mathcal{D}_{\mathcal{C}_{+}} \sqcup \mathcal{D}_{\mathcal{C}_{-}}$and $\mathcal{D}_{\mathcal{C}^{c}}^{c}=\mathcal{D}_{\mathcal{C}_{+}^{c}}^{c} \sqcup \mathcal{D}_{\mathcal{C}_{-}^{c}}^{c}$. 
Then we prove that

$$
\begin{aligned}
& \partial_{\lambda} \log \operatorname{det}_{F}\left(K(\lambda) K^{c}(\lambda)^{-1}\right) \\
& \quad=-\operatorname{Tr}\left((\mathcal{D}-\lambda)^{-1}-\left(\mathcal{D}_{\mathcal{C}}-\lambda\right)^{-1}-\left(\left(\mathcal{D}^{c}-\lambda\right)^{-1}-\left(\mathcal{D}_{\mathcal{C}^{c}}^{c}-\lambda\right)^{-1}\right)\right) .
\end{aligned}
$$

The operator in parentheses turns out to be trace class! In fact, the auxiliary model problem was introduced for exactly this reason: to overcome certain trace class issues, and the fact that the gluing problem on the model problem can be solved exactly [26]. The second remarkable property of $K(\lambda)$ and $K^{c}(\lambda)$ is that, using (4.2) and deriving formulas for the spectral invariants in terms of resolvents, we prove that for $\nu \in \mathbb{R}$ the spectral invariants can be expressed as

$$
\begin{aligned}
& \left.\frac{\operatorname{det}_{\zeta}\left(\mathcal{D}^{2}+\nu^{2}, \mathcal{D}_{\mathcal{C}}^{2}+\nu^{2}\right)}{\operatorname{det}_{\zeta}\left(\left(\mathcal{D}^{c}\right)^{2}+\nu^{2},\right.},\left(\mathcal{D}_{\mathcal{C}}^{c}\right)^{2}+\nu^{2}\right) \\
& \quad=C \cdot \operatorname{det}_{F}\left(K(i \nu) K^{c}(i \nu)^{-1}\right) \cdot \operatorname{det}_{F}\left(K(-i \nu) K^{c}(-i \nu)^{-1}\right),
\end{aligned}
$$

for a constant $C$, where $\operatorname{det}_{\zeta}\left(\mathcal{D}^{2}+\nu^{2}, \mathcal{D}_{\mathcal{C}}^{2}+\nu^{2}\right):=\operatorname{det}_{\zeta}\left(\mathcal{D}^{2}+\nu^{2}\right) \cdot\left(\operatorname{det}_{\zeta}\left(\mathcal{D}_{\mathcal{C}}^{2}+\nu^{2}\right)\right)^{-1}$ with a similar formula for the operators on the model cylinder, and

$$
\begin{aligned}
& \left.\eta \mathcal{D}, \mathcal{D}_{\mathcal{C}}\right) \\
=-\frac{1}{\pi i} & \left(\lim _{\nu \rightarrow \infty}\left(\log \operatorname{det}_{F}\left(K(i \nu) K^{c}(i \nu)^{-1}\right)-\log \operatorname{det}_{F}\left(K(-i \nu) K^{c}(-i \nu)^{-1}\right)\right)\right. \\
& \left.\quad-\lim _{\nu \rightarrow 0^{+}}\left(\log \operatorname{det}_{F}\left(K(i \nu) K^{c}(i \nu)^{-1}\right)-\log \operatorname{det}_{F}\left(K(-i \nu) K^{c}(-i \nu)^{-1}\right)\right)\right),
\end{aligned}
$$

where $\eta\left(\mathcal{D}, \mathcal{D}_{\mathcal{C}}\right):=\eta(\mathcal{D})-\eta\left(\mathcal{D}_{\mathcal{C}}\right)$. Third, as $\nu \rightarrow \pm \infty$, we prove that

$$
\begin{gathered}
\lim _{\nu \rightarrow \pm \infty} \frac{\operatorname{det}_{\zeta}\left(\mathcal{D}^{2}+\nu^{2}, \mathcal{D}_{\mathcal{C}}^{2}+\nu^{2}\right)}{\operatorname{det}_{\zeta}\left(\left(\mathcal{D}^{c}\right)^{2}+\nu^{2},\left(\mathcal{D}_{\mathcal{C}}^{c}\right)^{2}+\nu^{2}\right)}=1, \\
\lim _{\nu \rightarrow \pm \infty} \operatorname{det}_{F}\left(K(i \nu) K^{c}(i \nu)^{-1}\right)=a_{ \pm},
\end{gathered}
$$

where $a_{+}=1$ and $a_{-}=\operatorname{det}_{F} U$, and as $\nu \rightarrow 0^{+}$, we have

$$
\operatorname{det}_{F}\left(K( \pm i \nu) K^{c}( \pm i \nu)^{-1}\right)=( \pm \nu)^{h_{M}}(\operatorname{det} \mathcal{L})^{-1} \operatorname{det}_{F}\left(\frac{\operatorname{Id}+\widehat{U}}{2}\right)(1+o(1)) .
$$

Using the aforementioned properties, we can now prove the gluing formulas "in one shot"! For the $\zeta$-determinant, we first find the constant $C$ in (4.3). To do so, we take $\nu \rightarrow \infty$ on both sides of (4.3), and use the limits in (4.5) and (4.6) to obtain $C=\operatorname{det}_{F} U^{-1}=(-1)^{h_{M}} \cdot \operatorname{det}_{F} \widehat{U}^{-1}$. Substituting this value into (4.3), then using (4.7) and simplifying, we see that as $\nu \rightarrow 0^{+}$,

$$
\begin{aligned}
& \frac{\operatorname{det}_{\zeta}\left(\mathcal{D}^{2}+\nu^{2}, \mathcal{D}_{\mathcal{C}}^{2}+\nu^{2}\right)}{\operatorname{det}_{\zeta}\left(\left(\mathcal{D}^{c}\right)^{2}+\nu^{2},\left(\mathcal{D}_{\mathcal{C}}^{c}\right)^{2}+\nu^{2}\right)} \\
& =\nu^{2 h_{M}}(\operatorname{det} \mathcal{L})^{-2} \operatorname{det}_{F}\left(\frac{2 \operatorname{Id}+\widehat{U}+\widehat{U}^{-1}}{4}\right)(1+o(1)) .
\end{aligned}
$$

However, we prove that as $\nu \rightarrow 0^{+}$, the left-hand side has the asymptotics

$$
\frac{\operatorname{det}_{\zeta}\left(\mathcal{D}^{2}+\nu^{2}, \mathcal{D}_{\mathcal{C}}^{2}+\nu^{2}\right)}{\operatorname{det}_{\zeta}\left(\left(\mathcal{D}^{c}\right)^{2}+\nu^{2},\left(\mathcal{D}_{\mathcal{C}}^{c}\right)^{2}+\nu^{2}\right)}=\nu^{2 h_{M}} \frac{\operatorname{det}_{\zeta}\left(\mathcal{D}^{2}, \mathcal{D}_{\mathcal{C}}^{2}\right)}{\operatorname{det}_{\zeta}\left(\left(\mathcal{D}^{c}\right)^{2},\left(\mathcal{D}_{\mathcal{C}^{c}}^{c}\right)^{2}\right)}(1+o(1)) .
$$


Combining this expression with the previous equality, then setting $\nu=0$, we obtain

$$
\operatorname{det}_{\zeta}\left(\mathcal{D}^{2}, \mathcal{D}_{\mathcal{C}}^{2}\right)=\operatorname{det}_{\zeta}\left(\left(\mathcal{D}^{c}\right)^{2},\left(\mathcal{D}_{\mathcal{C}^{c}}^{c}\right)^{2}\right)(\operatorname{det} \mathcal{L})^{-2} \operatorname{det}_{F}\left(\frac{2 \operatorname{Id}+\widehat{U}+\widehat{U}^{-1}}{4}\right) .
$$

Finally, the main result of [26] tells us that $\operatorname{det}_{\zeta}\left(\left(\mathcal{D}^{c}\right)^{2},\left(\mathcal{D}_{\mathcal{C}^{c}}^{c}\right)^{2}\right)=2^{-\zeta_{D_{Y}^{2}}(0)-h_{Y}}$, which completes the proof for the $\zeta$-determinant.

Before proving the eta invariant formula, recall that if $f(t)$ is a smooth nonzero complex-valued function on an interval $[a, b]$, then the winding number $W(f) \in \mathbb{Z}$ of $f$ is defined by the equality

$$
\log f(b)-\log f(a)=\log f(b)-\log f(a)+2 \pi i W(f),
$$

where $\log f(t)$ is any continuous logarithm for $f(t)$ with $t \in[a, b]$ and Log denotes the principal value of the logarithm. In view of the limits (4.6) and (4.7), by definition of the winding number (4.8), we have, modulo $2 \pi i \mathbb{Z}$,

$$
\lim _{\nu \rightarrow \infty}\left(\log \operatorname{det}_{F}\left(K(i \nu) K^{c}(i \nu)^{-1}\right)-\log \operatorname{det}_{F}\left(K(-i \nu) K^{c}(-i \nu)^{-1}\right)\right) \equiv-\log \operatorname{det}_{F} U,
$$

where the integer defect is just the winding number of $\operatorname{det}_{F}\left(K(\lambda) K^{c}(\lambda)^{-1}\right)$ from $-i \infty$ to $i \infty$, and modulo $2 \pi i \mathbb{Z}$,

$$
\begin{aligned}
\lim _{\nu \rightarrow 0^{+}}\left(\log \operatorname{det}_{F}\left(K(i \nu) K^{c}(i \nu)^{-1}\right)-\log \operatorname{det}_{F}\left(K(-i \nu) K^{c}(-i \nu)^{-1}\right)\right) & \\
& \equiv \begin{cases}0, & h_{M} \text { even, } \\
-\pi i, & h_{M} \text { odd },\end{cases}
\end{aligned}
$$

where the integer defect is just the winding number of $\operatorname{det}_{F}\left(K(\lambda) K^{c}(\lambda)^{-1}\right)$ from $-i \nu$ to $i \nu$ for $\nu>0$ sufficiently small. Substituting these limits into (4.4) completes the proof for the eta invariant.

Acknowledgement. The authors thank Gerd Grubb and K. P. Wojciechowski for all their help and for taking a keen interest in our mathematical careers.

\section{REFERENCES}

1. M. F. Atiyah, V. K. Patodi, and I. M. Singer, Spectral asymmetry and Riemannian geometry. I, Math. Proc. Cambridge Philos. Soc. 77 (1975), 43-69. MR0397797 (53:1655a)

2. D. Bleecker and B. Booss-Bavnbek, Spectral invariants of operators of Dirac type on partitioned manifolds, Aspects of Boundary Problems in Analysis and Geometry, Birkhäuser, Boston, 2004, pp. 1-130. MR.2072498

3. J. Brüning and M. Lesch, On the $\eta$-invariant of certain nonlocal boundary value problems, Duke Math. J. 96 (1999), 425-468. MR.1666570 (99m:58180)

4. V. Bruneau, Fonctions zêta et êta en présence de spectre continu, C. R. Acad. Sci. Paris Sér. I Math. 323, no. 5 (1996), 475-480. MR1408979 (97j:58154)

5. U. Bunke, On the gluing formula for the $\eta$-invariant, J. Differential Geometry 18 (1995), 397-448. MR 1331973 (96c:58163)

6. D. Burghelea, L. Friedlander, and T. Kappeler, Mayer-Vietoris type formula for determinants of differential operators, J. Funct. Anal. 107 (1992), 34-65. MF.1165865 (93f:58242)

7. A.-P. Calderón, Boundary value problems for elliptic equations, Outlines Joint Sympos. Partial Differential Equations (Novosibirsk, 1963), Acad. Sci. USSR Siberian Branch, Moscow, 1963, pp. 303-304. MR0203254 (34:3107)

8. G. Carron, Déterminant relatif et la fonction Xi, Amer. J. Math. 124, no. 2 (2002), 307-352. MR1890995 (2003c:58023)

9. X. Dai and D. Freed, $\eta$-invariants and determinant lines, J. Math. Phys. 35 (1994), 5155-5195. MR1295462(96a:58204) 
10. R. Forman, Functional determinants and geometry, Invent. Math. 88 (1987), 447-493. MR0884797 (89b:58212)

11. G. Grubb, Trace expansions for pseudodifferential boundary problems for Dirac-type operators and more general systems, Ark. Math. 37 (1999), 45-86. MR1673426 (2000c:35265)

12. . Poles of zeta and eta functions for perturbations of the Atiyah-Patodi-Singer problem, Comm. Math. Phys. 215 (2001), 583-589. MR1810945 (2002a:58036)

13. Spectral boundary conditions for generalizations of Laplace and Dirac operators, Comm. Math. Phys. 240 (2003), 243-280. MR2004987(2004f:58030)

14. A. Hassell, Analytic surgery and analytic torsion, Comm. Anal. Geom. 6, no. 2 (1998), 255289. MR.1651417 (2000c:58061)

15. A. Hassell and S. Zelditch, Determinants of Laplacians in exterior domains, IMRN 18 (1999), 971-1004. MR1722360 (2001a:58045)

16. A. Hassell, R. R. Mazzeo, and R. B. Melrose, Analytic surgery and the accumulation of eigenvalues, Comm. Anal. Geom. 3 (1995), 115-222. MR1362650|(97f:58132)

17. A signature formula for manifolds with corners of codimension two, Topology $\mathbf{3 6}$, no. 5 (1997), 1055-1075. MR1445554 (98c:58163)

18. P. Kirk and M. Lesch, The eta invariant, Maslov index, and spectral flow for Dirac-type operators on manifolds with boundary, Forum Math. 16 (2004), 553-629. MR2044028 (2005b:58029)

19. Y. Lee, Burghelea-Friedlander-Kappeler's gluing formula for the zeta-determinant and its applications to the adiabatic decompositions of the zeta-determinant and the analytic torsion, Trans. Amer. Math. Soc. 355, no. 10 (2003), 4093-4110. MR1990576 (2004e:58058)

20. M. Lesch and K. P. Wojciechowski, On the $\eta$-invariant of generalized Atiyah-Patodi-Singer boundary value problems, Illinois J. Math. 40, no. 1 (1996), 30-46. MR.1386311 (97d:58194)

21. P. Loya, Dirac operators, boundary value problems, and the b-calculus, Contemp. Math. 366 (2005), 241-280.

22. P. Loya and J. Park, Decomposition of the $\zeta$-determinant for the Laplacian on manifolds with cylindrical end, Illinois J. Math. to appear.

23. - On the gluing problem for the spectral invariants of Dirac operators, Advances in Math. to appear.

24. - On the gluing problem for Dirac operators on manifolds with cylindrical ends, Preprint, 2004.

25. 2004.

26. The $\zeta$-determinant of generalized APS boundary problems over the cylinder, J. Phys. A. 37, no. 29 (2004), 7381-7392. MF.2078962

27. $ـ$ Asymptotics of $\zeta$-determinant of Dirac Laplacian under adiabatic process, In preparation.

28. R. Mazzeo and R. B. Melrose, Analytic surgery and the eta invariant, Geom. Funct. Anal. 5, no. 1 (1995), 14-75. MR1312019 (96a:58200)

29. R. Mazzeo and P. Piazza, Dirac operators, heat kernels and microlocal analysis. II. Analytic surgery, Rend. Mat. Appl. (7) 18, no. 2 (1998), 221-288. MR.1659838 (2000a:58065)

30. R. B. Melrose, The Atiyah-Patodi-Singer index theorem, A. K. Peters, Wellesley, 1993. MR1348401 (96g:58180)

31. W. Müller, Eta invariants and manifolds with boundary, J. Differential Geometry 40 (1994), 311-377. MR 1293657 (96c:58165)

32. - On the $L^{2}$-index of Dirac operators on manifolds with corners of codimension two. I, J. Differential Geometry 44 (1996), 97-177. MR1420351 (98b:58163)

33. - Relative zeta functions, relative determinants and scattering theory, Comm. Math. Phys. 192 (1998), 309-347. MR1617554 (99k:58189)

34. J. Park and K. P. Wojciechowski, Scattering theory and adiabatic decomposition of the $\zeta$ determinant of the Dirac Laplacian, Math. Res. Lett. 9, no. 1 (2002), 17-25. MR1892311 (2003c:58024)

35. - Adiabatic decomposition of the $\zeta$-determinant and Scattering theory, MPI Preprint, 2002.

36. D. B. Ray and I. M. Singer, R-torsion and the Laplacian on Riemannian manifolds, Advances in Math. 7 (1971), 145-210. MR0295381 (45:4447)

37. S. Scott, Zeta determinants on manifolds with boundary, J. Funct. Anal. 192, no. 1 (2002), 112-185. MR.1918493 (2003g:58051) 
38. S. Scott and K. P. Wojciechowski, The $\zeta$-determinant and Quillen determinant for a Dirac operator on a manifold with boundary, Geom. Funct. Anal. 10 (1999), 1202-1236. MR1800067 (2001k:58067)

39. R. T. Seeley, Topics in pseudo-differential operators, Pseudo-Diff. Operators (C.I.M.E., Stresa, 1968), 1969, pp. 167-305. MF 0259335 (41:3973)

40. I .M. Singer The eta invariant and the index, Mathematical aspects of string theory, World Scientific, Singapore, 1988, pp. 239-258. MR0915824

41. _ Families of Dirac operators with applications to physics, Astérisque, Numero Hors Serie, The mathematical heritage of Élie Cartan (Lyon, 1984), 1985, pp. 323-340. MR0837207 (88a:58192)

42. S. M. Vishik, Generalized Ray-Singer conjecture. I. A manifold with a smooth boundary, Comm. Math. Phys. 167, no. 1 (1995), 1-102. MR1316501 (96f:58184)

43. K. P. Wojciechowski, The additivity of the $\eta$-invariant. The case of a singular tangential operator, Comm. Math. Phys. 169 (1995), 315-327. MR1329198 (96k:58210)

44. The $\zeta$-determinant and the additivity of the $\eta$-invariant on the smooth, self-adjoint Grassmannian, Comm. Math. Phys. 201, no. 2 (1999), 423-444. MR.1682214|(2000f:58071)

Department of Mathematics, Binghamton University, Binghamton, New York 13902

E-mail address: paul@math.binghamton.edu

Mathematisches Institut, Universität Bonn, Beringstrasse 1, D-53115 Bonn, Germany

E-mail address: jpark@math.uni-bonn.de 